\title{
Le patrimoine littéraire : un enjeu de formation
}

\section{Brigitte Louichon}

\section{OpenEdition}

Journals

Édition électronique

URL : https://journals.openedition.org/trema/3285

DOI : $10.4000 /$ trema.3285

ISSN : 2107-0997

\section{Éditeur}

Faculté d'Éducation de l'université de Montpellier

\section{Édition imprimée}

Date de publication : 1 mai 2015

Pagination : 22 - 31

ISSN : 1167-315X

\section{Référence électronique}

Brigitte Louichon, «Le patrimoine littéraire : un enjeu de formation », Tréma [En ligne], 43 | 2015, mis en ligne le 25 juin 2015, consulté le 28 juin 2022. URL : http://journals.openedition.org/trema/3285 ; DOI : https://doi.org/10.4000/trema.3285

Ce document a été généré automatiquement le 29 septembre 2020.

Trema 


\title{
Le patrimoine littéraire : un enjeu de formation
}

\author{
Brigitte Louichon
}

1 L'un des piliers du socle commun de connaissances et de compétences s'intitule « culture humaniste ». Dans le paragraphe introductif, l'enseignant de lettres retrouve ce qui constitue historiquement et fondamentalement sa mission : « l'analyse et l'interprétation des textes » et « la fréquentation des œuvres littéraires ». En termes de connaissances, il convient de faire en sorte que les élèves connaissent « des textes majeurs de l'Antiquité » et les œuvres littéraires [...] majeures du patrimoine français, européen et mondial. En termes de capacités, les élèves doivent pouvoir «situer dans le temps [...] les œuvres littéraires». Enfin, en termes d'attitudes, la culture humaniste « donne à chacun l'envie d'avoir une vie culturelle personnelle, par la lecture [...]».

2 Le « socle commun » est paru en 2006 et les programmes qui ont suivi ont plus ou moins intégré les connaissances, capacités et attitudes dans les déclinaisons par niveaux et disciplines. Il est tout à fait notable que les expressions "patrimoine littéraire», « littérature patrimoniale », « œuvres du patrimoine », «textes patrimoniaux » etc. vont intégrer l'ensemble des programmes parus entre 2008 et 2012, de la maternelle au lycée. Ce phénomène est d'autant plus remarquable que le terme "patrimoine» était totalement absent des programmes précédents, lesquels, parlant des mêmes œuvres, les qualifiaient de "majeures», "de référence » ou "significatives». On en conviendra, qualifier une même œuvre de "patrimoniale» ou de «significative ", n'induit pas les mêmes effets de sens.

3 Je considère donc, de manière a priori sans doute un peu paradoxale, que le patrimoine littéraire est un objet scolaire nouveau. À ce titre, il doit être défini et réfléchi épistémologiquement et didactiquement en formation d'enseignants.

\section{Connotations}

4 Le terme "patrimoine » n'est ni neuf, ni anodin. Par delà son origine juridique, il se manifeste depuis les années 1970 par une capacité à s'étendre à des catégories d'objets de 
plus en plus divers. André Chastel, faisant l'histoire du mot et de la notion, indique : «En s'élargissant, la notion prend une valeur affective plus marquée pour désigner certaines conditions fondamentales de l'existence nationale, voire de l'existence humaine. Cette évolution ne fait peut-être que traduire le trouble de la conscience collective face à des menaces, plus ou moins précises, plus ou moins obscures, pour son intégrité ». L'enquête historique montre que l'acception contemporaine de la notion est consubstantielle de l'idée de caducité, de perte, d'usure, de disparition d'une part, et, d'autre part, de celle de valeur générale, commune, collective. Est donc patrimonial ce qui risque de disparaître et qui nous constitue collectivement.

5 La requalification massive, systématique, simultanée, du corpus scolaire littéraire classique ou de référence pose nécessairement question car elle conduit à infléchir sensiblement la représentation de la littérature, de ses usages et de son enseignement. On peut dégager quelques effets connotatifs.

6 Définie comme relevant du patrimoine culturel, la littérature est considérée comme un objet culturel comme les autres. C'est la continuation logique d'un affaiblissement en termes de positionnement hiérarchique et symbolique tant dans le champ social que dans le champ de l'enseignement. Il y a un brouillage des spécificités: les œuvres sont considérées comme des objets culturels et la lecture des œuvres devient une pratique culturelle, parmi d'autres. À l'école, l'introduction de la notion de culture humaniste paraît de même nature.

7 D'autre part, la littérature est de fait considérée comme menacée de disparition, affectée par ce sentiment de perte et d'effacement propre au patrimoine. Les œuvres littéraires doivent être préservées, menacées qu'elles sont par d'autres pratiques culturelles (le numérique, la musique, le cinéma...) ou d'autres lectures (la BD, la littérature jeunesse). C'est parce qu'elle est perçue comme relevant du passé, qu'elle est menacée par le présent. Notons que cette représentation va à l'encontre de celle de la littérature classique dont la valeur a toujours été du côté du renouvellement, et de sa capacité à parler du présent et au présent. L'œuvre classique n'a pas besoin d'être préservée, car comme le dit (par exemple) Philippe Sollers, « c'est un organisme en train de se composer et de se recomposer sans cesse ».

8 La préservation du patrimoine ne se justifie qu'en ce qu'il est doté de valeur collective. La littérature participe de la "culture commune » présente depuis 2002 dans le champ de l'école. La littérature patrimoniale est définie comme un ensemble d'objets patrimoniaux, de « lieux de mémoire » constitués par les textes. Cette représentation s'oppose à celle de la littérature comme construction historique et sociale, toujours en mouvement, ou d'une représentation de la littérature comme fondamentalement intertextuelle, ou encore d'une représentation de la littérature centrée sur le lecteur. On retrouve cette vision de la littérature dans les programmes pour l'école primaire de 2008 qui sous l'intitulé « littérature » ne renvoie qu'à une liste de textes, d'objets.

Enfin, la littérature comme patrimoine, est comme déconnectée du champ de la critique ou de l'histoire littéraires. Bien que venue du passé, la littérature patrimonialisée semble exister hors de l'histoire et de la glose. Elle devient une essence, une évidence qu'on ne questionne pas.

De fait, cette littérature patrimoniale constituée d'œuvres monumentales se prête assez bien aux objectifs évoqués du socle commun : connaître les œuvres et les situer dans le temps. 
11 Disons-le tout net, cette introduction massive du terme "patrimoine " dans les textes institutionnels amène à une vision réactionnaire de la littérature et de son enseignement. Face à cette situation, on peut envisager trois attitudes. La première consiste à ignorer cette dimension, à faire comme si l'usage des mots et les effets de sens n'existaient pas. La seconde consiste à faire en sorte que le mot disparaisse aussi vite qu'il est apparu, à la faveur d'une "refondation de l'école » qui refuse la dimension réactionnaire dont procède l'emploi de la du terme. La troisième consiste à tenter de définir la littérature patrimoniale pour ce qu'elle est, à partir de l'observation.

\section{Définition}

12 La très grande majorité des œuvres du passé est oubliée, disparue, hors d'usage. Personne ne lit plus ces œuvres, personne ne peut plus les lire. Au regard de la production, quelques rares œuvres, demeurent, rarement rééditées, disponibles sur les sites spécialisées, lues par les spécialistes ou les curieux. Enfin, une toute petite minorité d'œuvres est présente. Venues du passé, elles survivent et habitent notre présent. Elles sont les œuvres patrimoniales. En effet, le patrimoine, ce n'est pas le passé. C'est, à l'instar de la mémoire, le passé dans le présent, le passé présent. Pour ce qui concerne le patrimoine littéraire, cela signifie production passée et réception présente. Dès lors, observer et décrire la patrimonialité effective d'une œuvre consiste à observer et décrire les modalités de sa présence, de son actualité, c'est-à-dire l'ensemble des textes, des discours, des objets contemporains qu'elle génère, directement ou indirectement, lesquels constituent les preuves de son actualité et donc de sa patrimonialité. Je désigne l'ensemble de ces textes et discours comme des "objets sémiotiques secondaires » (dorénavant OSS) et je propose de classer ces objets en quatre catégories.

\section{1. Les adaptations}

On donnera à ce terme son sens le plus large, celui d'adaptation à un public donné et à un support donné et/ou à un genre donné.

Dans cette perspective, les éditions sont des adaptations. Une nouvelle édition d'une œuvre classique vise à s'adapter au plus près aux attentes supposées d'un lectorat ciblé (souvent scolaire ou universitaire). L'ajout d'un paratexte toujours différent, toujours renouvelé, même si le texte est conservé dans son originalité constitue une forme d'adaptation. La présence de ce même texte original sur des sites internet, dont les enjeux et les formes sont très divers, sur lesquels le texte est en lien avec ou à partir d'éléments différents, relève d'une forme d'adaptation du texte.

Dans cette perspective, la traduction participe aussi de l'adaptation de l'œuvre, adaptation à un lectorat étranger. De même, les « adaptations pour la jeunesse ", relevant d'une traduction intra-linguistique (ou réécriture), ou les éditions abrégées sont aussi adaptation à un lectorat en fonction de ses compétences lexiques; de même que les éditions en braille sont des adaptations à un public spécifique...

Enfin, j'inclus dans cette catégorie ce que l'on appelle parfois « adaptation », «traduction intersémiotique », « transmutation », «transposition » ou « transécriture » selon que l'on met l'accent sur le langage, le média, le genre ou l'activité. Ce sont les Madame Bovary en roman-photo... Les Misérables en comédie musicale ... Les Fables de La Fontaine en spectacle 
théâtral ... Les Contes de Perrault ou d'Andersen en dessin animé ... La Recherche du temps perdu en BD...

\section{2. Les hypertextes et les transfictions}

17 On le sait, la littérature se souvient d'elle-même et se réécrit toujours. Les œuvres patrimoniales sont constamment convoquées, et donc, réactualisées, re-présentifiées par les œuvres contemporaines.

18 Si l'on reprend la typologie des pratiques hypertextuelles bien connue de Gérard Genette, les textes générés le sont par transformation (dans le cas de la parodie, du travestissement ou de la transposition) ou par imitation (dans le cas du pastiche, de la charge ou de la forgerie).

19 Mais l'œuvre patrimoniale, plus que des hypertextes, génèrent souvent ce que Richard Saint-Gelais appellent des "fictions transfuges", des récits et des histoires qui font migrer un élément fictionnel (souvent le personnage), qui racontent autre chose à son propos. "L'hypertextualité, précise l'auteur, est une relation d'imitation et de transformation entre textes; la transfictionnalité, une relation de migration (avec la modification qui en résulte presque immanquablement) de données diégétiques ». La transfictionnalité permet de décrire et de comprendre des phénomènes d'autonomisation (au moins partielle) des personnages au regard de l'œuvre qui les a fait naitre. Ainsi, lorsque Tim Burton met en scène une Alice de 19 ans ou que Régis Loisel invente la première enfance de Peter Pan, ils ne réécrivent ni d'adaptent les œuvres originales, ils font migrer les personnages et un certain nombre de données fictionnelles dans une autre histoire.

\section{3. Les métatextes}

Ce sont les commentaires, les discours critiques, les discours historiographiques, les discours scolaires, les discours autobiographiques qui disent quelque chose du texte. Ce "quelque chose » varie suivant le cadre discursif, le genre textuel ou le type d'écrit mais la pratique relève globalement de la métatextualité. Plus un texte est commenté, analysé, expliqué, exposé, contextualisé, dans le cadre d'ouvrages critiques, historiques, d'anthologies, de manuels scolaires, d'ouvrages parascolaires, pédagogiques, sur les blogs, plus il est patrimonial.

\section{4. Les allusions}

21 Cette catégorie est présente aussi chez Genette qui l'analyse comme relevant d'une modalité de coprésence entre deux textes: «Un énoncé dont la pleine intelligence suppose la perception d'un rapport entre lui et un autre auquel renvoie nécessairement telle ou telle de ses inflexions, autrement non recevable». Autrement dit, le texte n'est pas énoncé, mais il est présent, nécessairement présent. Ce silence même est la marque de son actualité et de sa présence chez le lecteur. L'allusion relève ici exclusivement d'une économie stylistique. On pourrait presque parler de synecdoque. Un personnage, une phrase ou même un mot sont mis à la place d'une œuvre ou d'un texte, d'une histoire. À la limite, le titre de l'œuvre dans nombre de discours fonctionne de cette manière-là. 
L'allusion est donc purement dénotative. Pour le dire autrement, le Lecteur Modèle du texte dans lequel se trouve l'allusion connait le texte auquel il est fait allusion.

Cette typologie peut être discutée. Ainsi Christiane Connan-Pintado propose pour sa part le détournement comme catégorie englobant l'adaptation, la parodie, la réécritureréappropriation, la transposition, la variation et le mélange. En réalité, la typologie proposée n'a de valeur qu'heuristique. Elle permet de comprendre les relations qu'entretiennent les œuvres et, pour ce qui concerne la définition de l'œuvre patrimoniale, de décrire et d'observer les formes de la présence de l'œuvre.

Ainsi, l'œuvre patrimoniale se caractérise par sa présence effective et son actualité réelle. Celles-ci se réalisent et s'observent par les actualisations éditoriales de l'œuvre, par les adaptations qu'elle suscite, les nouveaux récits qu'elle génère, par les discours, les gloses, les analyses, les controverses qu'elle suscite et enfin par sa présence, cette fois immatérielle, dans la mémoire du lecteur.

24 Au final, il ne peut être question de considérer l'œuvre patrimoniale dans sa clôture et ses limites textuelles. On le sait, on ne fait jamais que "relire les classiques", il existe des livres dont on peut parler alors qu'on ne les a pas lus. L'œuvre patrimoniale n'existe pas hors «du nuage de discours" qu'elle génère. Cela doit nous amener à considérer simplement que l'œuvre patrimoniale, c'est l'œuvre escortée et augmentée de la multiplicité et la diversité des objets sémiotiques secondaires qu'elle génère.

\section{Propositions}

25 Si l'on accepte cette définition et déclinaison de l'œuvre patrimoniale, on est amené à envisager une approche didactique partiellement spécifique de la littérature patrimoniale. Celle-ci ne peut être exclusivement fondée sur les obstacles de type linguistique ou de référence que soulève nécessairement une œuvre datée. Elle doit s'ancrer dans la grande variété des objets sémiotiques secondaires générés par l'œuvre.

\section{1. Les OSS comme texte du lecteur}

Tout œuvre seconde d'adaptation, d'actualisation, de réécriture est œuvre de lecture et d'interprétation de l'œuvre première. À ce titre, elle peut être considérée comme un "texte de lecteur» au sens où l'entendent les didacticiens de la lecture littéraire travaillant à la notion de sujet lecteur. Ceux-ci proposent en effet un changement de paradigme pour penser la lecture littéraire en s'intéressant «à la reconfiguration du texte par le lecteur réel » et en observant, analysant « des modes de réalisation pluriels ». Ce changement de paradigme déplace le questionnement du lecteur virtuel au lecteur réel, et, par voie de conséquence, du «texte de l'œuvre» au «texte du lecteur». Si le texte du lecteur peut être constitué en données pour la recherche dans la mesure où il permet de comprendre ce qu'est la lecture, d'un point de vue praxéologique, il permet d'envisager une approche de la lecture scolaire fondée sur une ou des expériences de lecture antérieures dont les œuvres secondes sont les traces. Dans cette perspective, ces œuvres et l'œuvre originale entrent en dialogue et permettent une problématisation de la lecture de l'œuvre patrimoniale.

Ainsi, Gérard Langlade s'intéresse dans un article récent aux lectures cinématographiques de La Princesse de Clèves. «J'attends, écrit-il, de la créativité de ces lectures qu'elle brise 
l'effet inhibant de l'immobilité muséale dans laquelle l'œuvre est souvent maintenue, qu'elle stimule l'initiative interprétative des élèves et qu'elle donne accès à l'étrange fécondité d'un texte du XVIIe siècle qui interpelle sans cesse l'imaginaire, la sensibilité et le jugement de ses lecteurs». L'expression « lectures cinématographiques » met l'accent sur le fait qu'une adaptation est aussi une lecture. Certes, certains choix sont liés aux langages spécifiques de la littérature ou du cinéma mais d'autres procèdent de la manière dont le roman de Mme de La Fayette est lu. L'auteur montre ainsi, par exemple, que les quatre films proposent tous une réponse différente à la question du refus final de Mme de Clèves.

«Les quatre réponses que les lectures cinématographiques fournissent à la question du refus de la Princesse sont dans le roman [...] Chaque adaptation se construit en privilégiant l'une ou l'autre de ces raisons, en établissant une hiérarchie, voire en en supprimant une [...] ». Gérard Langlade conclut ainsi :

Je vois à cette lecture des lectures plusieurs intérêts didactiques. Prendre appui sur la créativité des lectures cinématographiques que ce roman génère donne à voir l'expansion interprétative d'un grand texte littéraire et fonde ce dernier en tant qu'œuvre patrimoniale : une cuvre du passé qui reste vivante en s'actualisant sans cesse dans de nouvelles lectures, de nouvelles interprétations, de nouvelles créations. Faire le lien entre la diversité des actualisations et les ferments d'affabulation de l'œuvre conduit à une démarche réflexive de retour au texte non pas pour y trouver une vérité établie mais pour y chercher les origines des constructions fictionnelles des lectures, ce qui conduit à appréhender une œuvre patrimoniale comme une cuvre - fabrique de lectures. Le jeu des dé/recontextualisations de l'œuvre invite également à s'intéresser, à rebours, au contexte initial singulier de son écriture: que doit - elle aux représentations sociales, aux idéologies, aux règles morales, à l'esthétique de son époque? Comment les lecteurs contemporains ont - ils répondu aux défis interprétatifs du roman? Quelle est la place de La Princesse de Clèves dans la production romanesque du XVIIe siècle et dans celle de son auteur?

On le voit, l'adaptation n'est pas un simple outil pédagogique. D'une part, les lectures cinématographiques sont considérées comme des actualisations de l'œuvre. Elles justifient la lecture de l'œuvre patrimoniale. La Princesse de Clèves n'est pas un classique poussiéreux dont la lecture est imposée par l'école au nom d'impératifs que l'on aurait oubliés ou que l'on ne comprendrait pas. L'école donne à lire une œuvre vivante. D'autre part, les adaptations, dans leurs diversités, mettent en exergue la dimension subjective et interprétative de la lecture. Elles empêchent le verrouillage herméneutique et vont à l'encontre d'une représentation de la lecture - a fortiori de la lecture scolaire - comme mise au jour d'un sens caché s'appuyant sur l'incompétence de l'élève et l'expertise professorale. Enfin, elles permettent et justifient le retour au texte et mettent en œuvre la dimension réflexive inhérente à la lecture littéraire.

\section{2. CEuvre patrimoniale et multimodalité}

Lire La Princesse de Clèves en faisant dialoguer œuvre littéraire et œuvres cinématographiques conduit à faire entrer dans la classe de français des objets médiatiques et sémiotiques multi-modaux. Le mode sémiotique se définit comme une ressource pour faire du sens (image, écriture, son, gestuelle, regard) et la multi-modalité est une combinatoire de modes. La bande-dessinée, l'album, le cinéma, le jeu vidéo relèvent de cet univers complexe. L'école est amenée à envisager la production/réception de sens d'objets multimodaux en termes de compétences à construire. 
Or, pour les enseignants de lettres, ces nouveaux objets, nouvelles approches, nouvelles compétences, semblent venir se surajouter à ce qui demeure : apprendre à lire et à écrire des textes. En outre et assez logiquement, on a le sentiment que plus la pratique des objets multi-modaux se développe dans l'espace privé et social, plus l'école doit se recentrer sur la lecture de la littérature. La seule solution consiste à mettre en lien la littérature et le multi-modal. La littérature patrimoniale est le lieu par excellence de cette rencontre nécessaire car consubstantielle de la patrimonialité. L'œuvre seconde multimodale est alors appréhendée, non seulement comme une lecture/interprétation de l'œuvre première mais aussi comme une adaptation à un langage différent, comme une production multimodale.

Je prendrai ici appui sur une étude comparée de quelques fables de La Fontaine en bandedessinée. Une question que l'on peut se poser concerne la manière dont les auteurs de bande-dessinée parviennent à maintenir le texte intégral lors même que les systèmes sémiotiques sont différents. Pour ne prendre qu'un exemple simple, la fable met en scène des animaux qui parlent. C'est une des données les plus centrales, les plus immédiates de l'œuvre de La Fontaine. L'alternance du récit et du dialogue donne aux saynètes une vivacité et une intensité remarquables.

Les dialogues sont le lieu de la caractérisation des personnages et les forces en jeu s'y expriment sans la médiation de la narration. La très grande majorité des fables propose donc du discours direct et du discours indirect inséré dans du récit.

Le langage écrit développe un système basé sur la ponctuation (deux points, tirets, guillemets) et l'attribution des discours, comme les modalités d'énonciation de ces discours, se font par l'intermédiaire de verbes introducteurs de dialogue plus ou moins riches sémantiquement (dire, crier, affirmer, s'exclamer etc.). Le langage de la BD, quant à lui, repose sur une alternance de récit (le récitatif dans le cartouche) et de discours direct (les bulles). Les appendices permettent l'attribution du discours direct à tel ou tel personnage en scène tandis que la graphie du discours (emploi de majuscules, de caractères gras) exprime les modalités de l'énonciation (colère, cri, murmure etc.).

Or, ces deux systèmes sont partiellement incompatibles. Si les discours prononcés par les personnages sont présents dans les deux cas, les modalités de leur attribution (et de manière plus secondaire de leur énonciation) s'excluent l'une l'autre. En toute logique, la présence de bulles et d'appendices devrait exclure la présence de forme comme « dit-il ». La question devient donc : comment les auteurs de BD se sortent-ils de cette contrainte du texte?

36 La même question peut être posée pour ce qui concerne la versification. La dimension versifiée des fables, qui articulent de manière très efficace des mètres différents et développent des systèmes de rimes parfois savants, est fondamentale. Visuellement, la fable est un bloc textuel, avec alinea à chaque fin de vers et majuscule à l'initiale. Cette dimension poétique est déterminante par rapport au statut de la fable comme genre scolaire et à son inscription dans les mémoires individuelles et collectives. Pour l'auteur de $\mathrm{BD}$, la question se pose donc de la transmutation de la matière visuelle et sonore. Que faire des rimes et des rythmes?

Enfin, la dimension apologétique de la fable mérite aussi que l'on s'y arrête. La fable se termine souvent par un énoncé de type gnomique, plus usuellement désigné sous le terme de «morale». Le récit et la morale se différencient fortement du point de vue linguistique dans le cas (fréquent) d'une énonciation finale et explicite. On bascule d'une 
narration au passé au présent de la généralité, des personnages à des formes indéfinies (on) ou des formes de personnes de l'interlocution (je, nous), d'un monde situé à une absence de référence. Il est aisé de décrire linguistiquement le glissement qui s'opère d'un système énonciatif à un autre. Mais qu'en est-il, que peut-il en être pour l'auteur de BD? Ces questions (récit et discours, versification, morale) renvoient à des traits caractéristiques de la fable comme genre, de même qu'elles constituent quelques-uns des obstacles à la compréhension pour les élèves. À ce titre, ce sont des entrées pédagogiques fréquentes. Le recours à la $\mathrm{BD}$ conserve cette perspective. Mais elle permet de mettre les élèves en situation de recherche, de problématiser la lecture en classe, de nécessiter un aller-retour permanent entre fable et BD, lecture de l'œuvre patrimoniale et lecture de l'œuvre contemporaine, de développer le savoir-lire La Fontaine et le savoir-lire la BD.

À ce titre, la mise en œuvre de situation de classe dans lesquelles œuvre patrimoniale (La Fontaine ou La Princesse de Clèves) et œuvres multimodales (BD ou cinéma) dialoguent permet aussi de travailler la question de la valeur et de développer des compétences critiques en termes d'évaluation et d'appréciation des œuvres. De fait, en regardant de près les choses, en confrontant l'œuvre source aux adaptations, par exemple dans les adaptations en $\mathrm{BD}$, on peut observer que les réponses des auteurs ne se valent pas. Certains tentent d'évacuer le problème tandis que d'autres trouvent des solutions ingénieuses, qui en retour donnent sens à la fable d'une manière nouvelle, inventive, contemporaine.

\section{3. Euvre patrimoniale et numérique}

Lecture, écriture, culture humaniste, histoire des arts... le domaine de l'enseignant de lettres semble se caractériser par une extension du domaine de compétences et d'intervention inversement proportionnelle à la réduction des horaires. Ajoutons donc le numérique!

41 L'une des caractéristiques fondamentales de la littérature patrimoniale est sa gratuité Parce qu'elle est libre de droits, l'œuvre peut être éditée, adaptée, récrite, parodiée, utilisée à moindre coût financier et à moindre risque juridique. Elle est donc partout, à commencer par internet.

Je voudrais ici évoquer rapidement trois aspects, trois modalités de rencontre entre patrimoine littéraire et numérique : le web comme espace de ressources, le web comme espace de partage, le numérique comme espace de création.

Le web donne accès au texte, aux manuscrits parfois, aux archives et à de nombreux OSS passés. Les éditions ou affiches de films récentes ou passées, les traductions, illustrations anciennes, les métatextes donnent à voir la présence et la patrimonialité de l'œuvre. Le web donne les moyens matériels d'historiciser, de mettre en perspective, d'interroger, de comparer des lectures, de travailler dans une perspective génétique.

Le web est aussi - et peut-être surtout pour les élèves - un espace de partage. On trouve profusion de témoignages de lecteurs sur des sites, des blogs ou des forums, sans compter les réseaux sociaux. Le site "fanfiction» (et «fanfiction.net» en français) est une véritable mine où l'on trouve témoignages, discussions et réécritures, y compris d'œuvres classiques, surtout en lien avec l'actualité médiatique (lorsqu'une adaptation cinématographique sort). Le web donne ainsi à voir la lecture, l'appétit de lire dont 
témoignent les jeunes lecteurs et le plaisir du dialogue dans des communautés virtuelles que soude le l'amour des livres et de l'écriture.

Enfin, le numérique permet des créer des objets nouveaux. L'un des plus intéressants est sans doute le livre augmenté, rendu possible par la disponibilité de l'œuvre que l'on peut intégrer à des applications comme subtext.com qui développe un espace collaboratif scolaire à partir de nombreuses fonctionnalités (annotations, commentaires, citations, liens ...). L'œuvre patrimoniale et ses OSS (adaptations et commentaires) peuvent ainsi être mis en lien et le dispositif permet de visualiser l'œuvre comme un espace qui transcende la clôture supposée de l'œuvre que l'objet-livre renforce.

\section{Conclusion}

La définition de l'œuvre patrimoniale comme génératrice d'OSS amène, on le voit, à contrecarrer les connotations passéistes, réactionnaires, réductrices et timorées que l'usage du terme " patrimoine " génère.

Le patrimoine littéraire que l'école doit transmettre aux élèves et qui participe de la culture humaniste doit être considérée pour ce qu'elle est d'abord: une littérature habitant notre présent, nourrissant de manière toujours renouvelée les créations contemporaines les plus diverses, présente dans nos vies et sur les écrans de notre époque. Il convient de noter que le web permet, en contrepoint, un recours aux archives et à l'historicisation. La didactisation du patrimoine littéraire doit relever d'une conception de l'œuvre comme machine à produire, dans le temps et dans l'espace, des lectures, des interprétations, des créations.

Ce faisant, la littérature participe pleinement d'une culture humaniste qui nécessite de nouer les apports disciplinaires de manière effective. Ce faisant, l'enseignant de lettres apprend aux élèves à « situer dans le temps [...] les œuvres littéraires » et à « les mettre en relation avec des faits historiques ou culturels utiles à leur compréhension ». Mais il leur permet également «de faire la distinction entre produits de consommation culturelle et œuvres d'art ", « d'avoir une approche sensible de la réalité », « de mobiliser leurs connaissances pour donner du sens à l'actualité » (socle commun, 2006).

\section{RÉSUMÉS}

La connaissance, la fréquentation et l'appropriation des œuvres littéraires patrimoniales est l'un des enjeux de la culture humaniste. Cet article propose de discuter et de définir la notion d'œuvre patrimoniale ou de littérature patrimoniale. De fait, les connotations liées à cette expression, importée du domaine de l'histoire de l'art, conduisent à une vision passéiste de la littérature et de son enseignement. Nous posons, au contraire, qu'une œuvre patrimoniale est production passée et réception présente. La présence de l'œuvre est attestée par les objets sémiotiques secondaires (OSS) qu'elle génère et que l'on peut classer en 4 catégories: les adaptations, les hypertextes et transfictions, les métatextes et les allusions. À partir de cette définition, on propose une didactique de l'œuvre patrimoniale que l'on considère comme l'œuvre 
originale et ses OSS. Quelques exemples d'utilisations didactiques des OSS, autour de la multimodalité et du numérique, sont proposés.

Knowledge, attendance and appropriation of literary heritage is one of the issues of humanistic culture. This paper proposes to discuss and define the notion of heritage literature. Actually, the connotations associated with the term, imported from the field of art history, lead to an outdated vision of literature and literature education. We assume, on a contrary, that a heritage work is bothpast production and present reception. The workgenerates "secondary semiotic objects" (OSS) that can be classified into 4 categories: adaptations, hypertexts and transfictions, metatexts and allusions. Some examples of educational uses of OSS, around multimodality and digital, are available.

\section{INDEX}

Mots-clés : didactique de l'œuvre patrimoniale, objets sémiotiques secondaires (OSS), patrimoine littéraire

Keywords : didactics of the patrimonial work, heritage littérature, "secondary semiotic objects" (OSS)

\section{AUTEUR}

\section{BRIGITTE LOUICHON}

Faculté d'Éducation - Université de Montpellier, ESPE-LR, LIRDEF (EA 3749) 